\title{
REFLEXIONES SOBRE LA ACTUACION DE LOS PRINCIPIOS DEL DERECHO DEL TRABAJO
}

\section{(A PARTIR DEL CASO DEL HORARIO DE VERANO)}

Javier Neves Mujica

En este cnsayo voy a analizar el caso de la suspensión del horario de verano decretada por el actual gobierno para $1986-\mathrm{y}$ repetida luego para 1987-, con la intención de reflexionar a partir de aquél sobre las posibles actuaciones que caben a los principios del Derecho del Trabajo ante las situaciones concretas.

Dada la extensión y complejidad de este solo tema, que desde mi punto de vista constituye una de las cuestiones fundamentales de la rama jurídica que trato, tendré que limitarme al estudio de los aspectos del caso vinculados a los principios laborales, dejando simplemente anotados los otros numerosos e importantes problemas que también se plantean en él. Sin embargo, con el objeto de enriquecer el estudio, en el tratamiento del tema seleccionado, no me restringiré al análisis de los hechos tal como ocurrieron, sino que plantearé diversas situaciones que también hubieran podido producirse, para comprobar la operación de los principios en cada una de las hipótesis.

En definitiva, mi intención es que los planteamientos que formularé sobre el tema de los principios del Derecho del Trabajo a propósito del caso del horario de verano, resulten igualmente aplicables a cualquier otra situación en la que concurran similares elementos.

\section{LAS NORMAS IMPLICADAS EN EL CASO}

El eje del caso lo constituye la suspensión del horario de verano para los trabajadores de las empresas estatales y la administración pública, dispuesta por el gobierno sucesivamente en 1986 y 
1987. Partiendo de este hecho debo, en primer lugar, reseñar las normas que crearon el derecho de los mencionados trabajadores. para ver luego las que lo desconocieron.

En este punto hay algunas normas comunes a los trabajadores de las empresas estatales y de la administración pública y otras que son exclusivas de uno de a mbos grupos.

\subsection{Las normas que crearon el derecho}

\subsubsection{Vormas Comumes}

a. La Ley 9049 (13.02.40) establecio un horario de verano para los empleados públicos y particulares de la costa, en virtud del cual deberia haber un intervalo de tres horas y media entre la labor de la mañana y la de la tarde. No se afectaba la duración de la jornada, que ya entonces era de 8 horas.

b. Varias normas transitorias dictadas para enfrentar el déficit de petróleo crudo y productos derivados, surgido a raíz de la crisis del mercado internacional, crearon los horarios corridos y escalonados para todas las actividades públicas y privadas (1). Estas disposiciones fijaban diversos horarios de inicio de las labores según las actividades, sin modificar la duración de la jornada, que seguía siendo de 8 horas, salvo para quienes habian pactado jornadas menores.

\subsubsection{Normas exclusivas de los trabajadores de las empresas estatales (2)}

a. En virtud de diversas normas, los trabajadores bancarios habian obtenido una jornada inferior a la ordinaria

(1) Decreto Ley 20509 (15.01.74), reglamentado por Resolución Suprema 010-74-INAP (15.02.74); Resolución Suprema 070-74-INAP-DNP (18. 12.71), especifica para el verano; Decreto Supremo 004-74-TR (30.04. 74 ), que garantizaba el mantenimiento de los derechos adquiridos, etc.

(2) Aqui me referiré únicamente al caso de la banca, por ser bastante representativo del conjunto y contener el primer reclamo que fue resuelto por el Poder Juclicial. 
paia los meses de verano: de 8 a 2.36 horas para los empleados y de 7.45 a 2.36 horas para los obreros. Tal era el mandato establecido por convenios colectivos y resoluciones administrativas vigentes para dicho sector. Entre éstos es particularmente importante la Resolución Directoral del 25.6.52 que se remite a los contratos y la costumbre para determinar la jornada de trabajo.

1.1.3 Normas exchasivas de los trabajadores de la Administración Pública

a. Los Decretos Leyes 17082 (24.20.68) y 17372 (14.01.69, modificado por el Decreto Ley 20230 del 27.11. 73) fijaron un horario de trabajo de 7.45 a 13.30 horas de lunes a viernes para los empleados del gobierno central, durante el verano. en la costa. y en la sierra y selva. respectivamente. En este caso. en definitiva. se estableció también una jornada de trabajo menor a la ordinaria.

En resumen, las normas comunes conllevaban modificaciones al horario de trabajo (establecimiento de intervalos o cambio en las horas de ingreso), sin alterar la jornada misma (número de horas de labor por dia). En cambio. las disposiciones exclusivas. tanto para los trabajadores de las empresas estatales como de la administración pública. sí significaron el establecimiento de una jornada reducida de labores: de 6.36 horas para los empleados y de 6.51 horas para los obreros en la banca. y de 5.45 horas en la administración pública.

\subsection{Las normas que suspendieron el derecho}

a. El Decreto Supremo 107-85-PCM (28.12.85), dispuso que entre el $1^{\circ}$ de enero y al 31 de marzo de 1986, en las empresas estatales y la administración pública. el horario de trabajo sería de 7.45 a 15.45 horas, incluidas 30 minutos para refrigerio.

b. El Decreto Supremo 068-86-PCM (26.3.86), repetía la norma anterior para el verano de 1987.

Respecto de ambas disposiciones, cabe señalar que, al igual que las mencionadas en los puntos 1.1 .2 y 1.1.3., modificaron la 
jornada de trabajo, en este caso en sentido contrario: extendieron a 8 horas lo que estaba fijado en alrededor de 6. De otro lado, ambos decretos se acogieron al art. 211.20 de la Constitución, que autoriza al Presidente de la República a dictar medidas extraordinarias en materia económica y financiera, cuando así lo requiere el interés nacional y con cargo a dar cuenta al Congreso. Sobre este punto volveré después.

\subsection{La disposición constitucional sobre la jornada de trabajo}

He precisado en los puntos anteriores que el problema planteado en este caso, es el de duración de la jornada de trabajo durante el verano, que fue primero rebajada de las 8 horas y luego restablecida a esa cantidad.

Por tal razón, considero que la primera tarea debe consistir en analizar la disposición constitucional referida a la jornada de trabajo. El art. 44, que en su primer párrafo es el pertinente, señala lo siguiente:

"La jornada ordinaria de trabajo es de ocho horas diarias y de cuarenta y ocho horas semanales. Puede reducirse por convenio colectivo o por ley".

Hay que determinar cuál es el "contenido esencial" del citado art. 44 (3). Al respecto, creo que caben dos interpretaciones: nes:

a. Podría sostenerse que la Constitución fija una jornada máxima de trabajo $y$, al permitir expresamente sólo su reducción, marca una tendencia progresiva en virtud de la cual, una vez operada la disminución, no se permitiría la posterior reampliación.

(3) El Tribunal Constitucional español ha establecido que el "contenido esencial". son "aquellas facultades o posibilidades de actuación necesarias para que el derecho sea recognoscible como pertinente al tipo descrito" y "aquella parte del contenido del derecho que es absolutamente necesaria para que los intereses jurrdicamente protegibles, que dan vida al derecho, resulten real, concreta y efectivamente protegidos" (Senten. cia $N^{\circ} 11 / 81$ del 8.4.81. Recurso de inconstitucionalidad No. 192180). Ver: "Sentencias del Tribunal Constitucional sobre Derecho del Trabajo", Madrid, Fundación Friedrich Ebert, 1984. 
Tal sería la conclusión a que se arribaría si se analizara el texto desde la teoría de la irreversibilidad de las normas laborales (4). Esta, según Sala Franco, considera imposible que una norma posterior empeore las condiciones reguladas por aquella que deroga. En definitiva, se impediría que las normas pudieran tener un contenido regresivo.

La aplicación de esta teoría al caso que estoy tratando, supondría la finalización del debate: de ninguna manera podrían ser válidos los decretos ni norma alguna que restablecieran un régimen laboral peyorativo en perjuicio de los trabajadores que ya disfrutaban del mayor beneficio y ni siquiera de los nuevos.

Considero que esta teoria, si bien recoge una aspiración esencial en la normativa laboral, cual es el permanente mejoramiento de los derechos de los trabajadores, introduce una rigidez excesiva, que dejaría un escasísimo margen para la ejecución de las plurales políticas laborales que caben legítimamente dentro del modelo constitucional (5).

b. La segunda interpretación consistiría en afirmar que el texto ha establecido una jornada máxima, señalando expresamente la posibilidad de la reducción para descartar también expresamente la de la ampliación más allá del límite de las 8 horas.

Desde esta perspectiva, sería compatible con la disposición constitucional no sólo la norma que redujera la duración de la jor-

(4) Sala Franco, Tomás, "El principio de la condición más beneficiosa”, en Revista de Política Social No 114, Madrid.

(5) La teoría opuesta, la de la modernidad, me parece aún más inaceptable en materia laboral, por la excesiva flexibilidad que permite. En aplicación de ésta, la nueva norma tendría aplicación inmediata, extinguiendo los derechos reconocidos por la antigua, como ocurre en el Derecho Civil (art. III del Título Preliminar del Código Civil). Pienso que la teoría que concilia mejor los intereses de los trabajadores con la libertad de plasmación de diversas políticas, es la de los derechos adquiridos. En virtud de ésta, cabría dictar una nueva norma regresiva, pero ella no afectaría a los trabajadores que ya habían adquirido el derecho al amparo de la anterior. Sobre el punto ver: Sala Franco, op. cit. y Ojeda Avilés, Antonio, "El principio de condición más beneficiosa", en Revista de Politica Social No 134 , Madrid. 
nada por debajo de las 8 horas, sino también la que la volviera a ampliar, siempre que no fijara una duración mayor a la anotada. En todo caso. naturalmente, habria que tener en cuenta la jerarquia de una y otra norma. para no afectar lo dispuesto por el art. 87 de la Constitución.

Es esta interpretación la que ofrece mayores posibilidades de a nálisis, dado que actuarán distintos principios según los tipos de normas que hubieran ordenado la disminución y la reampliación. De esto voy a ocuparme en el punto siguiente.

Antes de pasar al próximo tema. quiero expresar que ambas interpretaciones tienen en común, el considerar al art. 44 de la Constitución como una norma de derecho necesario relativo. esto es, una disposición que fija un beneficio minimo para los trabajadores, que puede ser mejorado por otras (6).

\section{LAS DIVERSAS HIPOTESIS DE TRABAJO}

Al empezar adverti que no me limitaria al análisis del caso tal cual ocurrió. sino que plantearia las diversas hipótesis posibles de haber sucedido para ver en cada una de ellas el juego de los principios laborales.

El trasfondo común a todas las hipótesis es el siguiente: hubo originalmente una jornada de 8 horas que fue reducida por convenios colectivos y resoluciones administrativas en el caso de las empresas estatales, y por ley en el de la administración pública, en alrededor de un par de horas para el verano. Se trata justamente del ejercicio de las dos posibilidades de disminución que admite la Constitución: ley o convenio colectivo.

Esta jornada reducida fue posteriormente reampliada hasta el limite de las 8 horas que fija la Constitución. en virtud del manda-

(6) Luis Enrique de la Villa Gil distingue entre las normas de derecho necesario absoluto que crean derechos indispensables hacia arriba o hacia abajo, las de derecho necesario relativo que crean derechos indisponibles hacia abajo pero disponibles hacia arriba y las de derecho dispositivo que crean derechos disponibles, en un todo. Ver: "El principio de la irrenunciabilidad de los derechos laborales", en Revista de Política Social No 85, Madrid. 
to de dos decretos, uno aplicable para el verano de 1986 y el otro para el de 1987.

Quicro a continuación, partiendo de la incuestionable validez de las normas de reducción de la jornada, plantear interrogaciones acerca de la validez de las normas de reampliación de la misma. Para estos efectos voy a tratar primero el caso en su desenvolvimiento real y luego las diversas hipótesis posibles.

\subsection{La inadmisibilidad de los decretos de urgencia en este campo}

Lo que efectivamente ocurrió fue que las normas de reampliación de la jornada eran dos decretos dictados al amparo del art. 211.20 de la Constitución, es decir, de los llamados decretos de urgencia.

Sin entrar al análisis del papel de estos decretos en nuestro ordenamiento jurídico, que es un tema ajeno al que me he propuesto abordar, quiero señalar únicamente que en nuestro pais se admite hoy dia que tales decretos tienen fuerza de ley ( 7 ), por lo que podrían válidamente suspender la vigencia de una ley (8). Sin embargo, estas normas tienen como requisitos para su expedición, el constituir medidas extraordinarias y referirse a materia económica y financiera. En definitiva, están condicionadas, como sosticne Alberto Predieri (9), a un supuesto de hecho: urgente y extraordinaria necesidad y por una competencia limitada: sólo las materias autorizadas por la Constitución. Adicionalmente, están sujetas al control posterior del Congreso.

(7) Analógamente a lo que ocurre en atros ordenamientos, como el español y el italiano, en los que el nuestro parece inspirarse, aunque lo haga de modo muy defectuoso.

(8) Más difícil es admitirlo en el caso de un convenio colectivo. Planteo este tema más adelante, en el punto 2.3.1.

(9) "El sistema de las fuentes del Derecho", en la Constitución Española de 1978 , estudio sistemático dirigido por los profesores Albertò Predieri y E. García de Enterria, Madrid, Editorial Civitas S.A., 1984. 
Garcia de Enterría y Fernández (10) dicen respecto del más preciso texto de la Constitución española, que el presupuesto habilitante es "la existencia de una situación de necesidad, cuya excepcionalidad quiere subrayarse mediante su adjetivación de extraordinaria, esto es, inusual e imprevisible, y urgente, es decir, no susceptible de ser afrontada a través del procedimiento legislativo, ni siquiera por el procedimiento de urgencia que prevén los Reglamentos de la Cámaras".

En este caso, creo que no se puede considerar extraordinaria una disposición que suspende la vigencia de una norma durante todo el término en que ésta debía aplicarse: las leyes y convenios colectivos que fijaban la jornada reducida regían sólo para el verano, por lo que una disposición que las sustituyera para todo ese periodo pasaria a constituirse en ordinaria. Incluso si se llegara a sostener que lo extraordinario estaría en la suspensión por un solo verano, la reiteración de la medida por dos años consecutivos, como sucedió en los hechos, evidenciaría su pretensión ordinaria.

En cuanto a la materia, pienso que en este caso es notorio el carácter laboral de las disposiciones que comento, en tanto afectan directamente la vigencia de derechos reconocidos expresamente por la legislación a los trabajadores. De otro lado, es criterio admitido que las normas de excepción se interpretan restrictivamente, por lo que debe entenderse por materia económica y financiera lo más estrechamente vinculado con el manejo presupuestario.

\subsection{Caso de reducción de la jornada dispuesta por ley}

Esta es la situación que se produjo en el caso de los trabajadores de la administración pública. Estos, como se ha señalado antes. habían obtenido una jornada reducida para el verano en virtud de disposiciones legales. Quiero en este caso plantear dos hipótesis acerca de una eventual reampliación ordenada por normas distintas.

(10) García de Enterría, Eduardo y Tomás Ramón Fernández, Curso de Derecho Administrativo, Madrid, Editorial Civitas S.A., 1984. 


\subsubsection{IItpótesis l: reampliación dispuesta por lev}

Aqui habría una ley 1 que fijaba el derecho a una jornada de 5.45 horas durante el verano y una ley 2 que extendia dicha jornada hasta las 8 horas. La ley 2 , de conformidad con el art. I del Título Preliminar del Código Civil, sería derogatoria de la Ley 1. El acto mismo de la derogación es incuestionable en nuestro ordenamiento. Sólo podría ser objetado si se le enfocara descle la teoría de la irreversibilidad de las normas laborales antes expuesta y descartada.

Siendo inatacable la derogación misma, cabe preguntarse únicamente por sus efectos. Podría ser que la ley 2 rigiera para todos los trabajadores, antiguos y nuevos, como ocurriría si acogiéramos la teoría de la modernidad; o que se aplicara sólo a los nuevos, respetando en el caso de los antiguos el derecho adquirido, conforme a la teoría del mismo nombre, ambas también reseñadas antes.

Ya he señalado mi preferencia por la última teoria, que considero más acorde con el carácter protector del Derecho del Trabajo. Quiero añadir aqui que, según de la Villa (11), para que un derecho pueda considerarse adquirido deben concurrir tres elementos, que en este caso están manifiestamente presentes:

1. Constitución de la relación juridica.

2. Producción del supuesto de hecho.

3. Ejercicio del derecho o posibilidad de su ejercicio.

Si se aceptara que los trabajadores antiguos tendrian derecho a conservar su ventaja respecto de la nueva norma peyorativa, el principio que se estaría acogiendo sería el de la condición más beneficiosa. Este actúa justamente, como señala Ojeda Avilés (12), en los supuestos de derogación y sucesión normativa, como el de la hipótesis propuesta, para garantizar "la conservación de los tratamientos obtenidos por aplicación de la normativa anterior si son

(11) Instituciones del Derecho del Trabajo, Madrid, Editorial CEURA, 1985.

(12) Op. cit. 
más beneficiosos o no se contemplan por la normativa sustituyente".

Debo agregar, por último, que este principio, tanto como cualquier otro, no requiere para su aplicación de la recepción normativa (13). Lo común es que los principios actúen válidamente aunque la normativa no los recoja expresamente, lo que sin embargo, ocurre con cierta frecuencia. Sólo una expresa norma en contrario - cuya legitimidad estaría sujeta a su verdadera excepcionalidadpodría impedir su actuación (14).

\subsubsection{Hipótesis 2: reampliación dispuesta por convenio colectivo}

Se tendría una ley que disminuiría la jornada por el verano y un convenio colectivo que la reampliaría. En este caso, la disposición del convenio colectivo que ordenara tal cosa sería nula, por contener una renuncia de derechos.

La Constitución establece en su art. 57, primer párrafo que los derechos reconocidos a los trabajadores son irrenunciables $\mathbf{y}$ pena con la nulidad todo pacto en contrario. En lo más específicamente referido a los convenios colectivos, el art. 48, primer párrafo, del Decreto Supremo 006-71-TR califica de nulos a los convenios colectivos y resoluciones administrativas que declaren, en perjuicio de los trabajadores, menores derechos que los establecidos por las leyes y sus reglamentos.

La renuncia es definitiva por de la Villa (15), como un "negocio jurídico unilateral que determina el abandono irrevocable de un derecho dentro de los límites establecidos por el ordenamiento". Este es el acto que se produciría en la hipótesis que planteo. $Y$ sería nulo, por afectar un derecho mínimo emanado de una nor-

(13) Esto no ocurre en el caso de la retroactividad benigna de las normas laborales (art. 187 de la Constitución) que es más bien la excepción a un principio que es la irretroactividad. Aquí se requeriría de norma expresa.

(14) Al respecto ver: Plá Rodríguez, Américo, Los principios del Derecho del Trabajo, Buenos Aires, Ediciones Depalma, 1978.

(15) "El principio de la irrenunciabilidad de los derechos laborales". 
ma de derecho necesario relativo - como es la ley que establece la jornada reducida-, esto es, con palabras de Sala Franco (16) imperativa a efectos de su peyoración y dispositiva a efectos de su mejora.

El principio de irrenunciabilidad, que es también la regla general en materia laboral, admite sin embargo excepciones. Resultarían renunciables, siguiendo la gradación normativa establecida por de la Villa que antes expuse, los derechos creados por normas de derecho necesario relativo en su parte dispositiva y de derecho dispositivo. Tal sería el caso, según el citado autor, de los derechos nacidos por encima de los minimos por via contractual individual y también los nacidos de costumbre (excepto la costumbre lla mada por una norma).

Finalmente quiero señalar que no sólo los derechos proclamados por ley -con el carácter descrito-son irrenunciables, sino también los creados por cualquier otra fuente del derecho, en particular, los convenios colectivos (17).

2.3. Caso de reducción de la jornada dispuesta por convenio colectivo

En este punto voy a hacer lo propio que en el 2.2., pero no sobre la base de una reducción de la jornada dispuesta por ley, sino por convenio colectivo, tal como ocurrió en el caso de los trabajadores bancarios que he escogido como representativo de las empresas estatales. La hipótesis serán las mismas.

\subsubsection{Hipótesis 1: reampliación dispuesta por lev.}

Se produciria esta situación si originalmente un convenio colectivo hubiera dispuesto la reducción de la jornada, la misma que luego se hubiera reampliado por mandato de la ley.

(16) Op. cit.

(17) El Decreto Supremo 006-71-TR dispone en su art. 43 que los convenios colectivos no pueden ser dejados sin efecto por acción individual ni por contrato individual. 
Al respecto considero, en primero lugar, que la ley no debe intervenir para regular una materia que ha sido objeto de tratamiento por un convenio colectivo. Entre la ley y el convenio colectivo existe, como señala Predreri (18), una relación de competencia, esto es, un reparto en función de las materias, dado que ambas normas se ubican en el mismo nivel de la jerarquía (19). Si bien el convenio colectivo está sujeto a la legalidad, ésta a su vez está obligada a respetar la autonomía colectiva, en un doble juego de controles que garantiza la Constitución y el Convenio 87 de la Organización Internacional del Trabajo para libertad sindical. Creo que una vez regulada una materia por convenio colectivo sólo podría admitirse la posterior intervención de una ley para mejorar lo pactado. La dación de una ley peyorativa, en mi opinión, resultaría aceptable en el excepcional caso de un imprescindible interés nacional y en forma provisional (20).

La ley y el convenio colectivo son dos normas del mismo nivel pero de distinto origen, razón por la cual la ley posterior en este caso no derogaría al convenio colectivo anterior. Ambas normas continuarían vigentes, regulando la misma situación con contenidos divergentes. El ámbito de regulación de las citadas normas no sería idéntico, sino sólo parcialmente coincidente, dadas las distintas esferas de aplicación de una ley y un convenio colectivo, por lo que habria entre ellas - con palabras de Martín Valverde (21)concurrencia conflictiva y no colisión opositiva. La cuestión, en definitiva se resolvería no con la derogación sino con el desplazamiento de una de las normas. En este supuesto operaría el principio de la norma más favorable, para seleccionar la norma aplicable al caso en función de st: mejor contenido para los trabajadores, que sería el convenio colectivo.

(18) Op. cit.

(19) En el nivel llamado primario, en el que se encuentran las normas con fuerza de ley, carácter que la Constitución reconoce a los convenios colectivos (art. 54).

(20) Análogamente, a lo que permitiría en materia no laboral, el art. 211.20 de la Constitución, si fuera utilizado correctamente.

(21) Martín Valverde, Antonio, "Concurrencia y articulación de normas la. borales", en Revista de Política Social No 119, Madrid. 
Este principio, según Alonso Oled (22) detcrmina que la norma a aplicar es la más favorable para el trabajador con independencia de su rango, que la postergada sigue formando parte del ordenamiento y que su exclusión se restringe al caso concreto. He sostenido en otro trabajo (23), que el principio en mención actúa - dentro de los márgenes señalados- en los siguientes cinco supuestos: entre normas estatales, entre normas estatales y convenios colectivos, entre convenios colectivos, entre normas nacionales e internacionales y entre normas internacionales.

\subsubsection{Hipótesis 2: reampliación dispuesta por convenio colectivo}

Aquí se tendria un convenio colectivo 1 que dispondría la disminución de la jornada y un convenio colectivo 2 que la reampliaría. Al respecto se podrían producir dos situaciones: 1) si los ámbitos de regulación de ambos convenios colectivos fueran exactos, operaría la derogación. y 2) si fueran sólo parcialmente coincidentes, operaría al desplazamiento.

En el primer caso, se aplicaría por analogía la derogación entre leyes, antes comentada. Y los problemas serían similares: efectos para todos o sólo para los nuevos, según se acogiera la teoría de la modernidad o de los derechos adquiridos, respectivamente. Pero habría un elemento adicional importante para optar en este caso por la última teoría: la doctrina no admite que un convenio colectivo posterior pueda modificar los derechos laborales normados para los trabajadores de anteriores convenios colectivos, como ocurriría en el caso planteado (24).

En el segundo caso, resultaría pertinente el principjo de norma más favorable, tal como lo he descrito antes. Esta situación de conflicto entre convenios colectivos, que no se resuelve con la derogación, no es factible en nuestro ordenamiento, por la ausencia

(22) Alonso Olea, Manuel, Derecho del Trabajo, Madrid, Universidad de Madrid, Facultad de Derecho, 1981.

(23) "Normas y principios del Derecho del Trabajo", en "Themis", Revista de Derecho, Segunda Fpoca, N 6 , Lima, 1987.

(24) Aunque algunos autores sostienen lo contrario, fundándose en el predominio del interés colectivo sobre el individual. Ver: de la Villa, op. cit. 
de la llamada contratación articulada, que permite el ensamblaje de convenios colectivos de diversos niveles, caso en el que podrían producirse las incompatibilidades (25).

\section{BREVE REFERENCIA A LA ACCIOV DE AMPARO INTERPUESTA POR LOS BANCARIOS}

No puedo, por último, dejar de mencionar y comentar, aunque sea sucintamente, el hecho de que los trabajadores afectados, representados por sus respectivas organizaciones, interpusieron sendas acciones de amparo contra la violación de su derecho a una jornada reducida de labores durante el verano. Voy a referirme a continuación, sólo al proceso iniciado por los trabajadores bancarios, por ser el primero que fue resuelto favorablemente para los accionantes. En el análisis tomaré en cuenta únicamente los aspectos netamente laborales contenidos en las tres sentencias emitidas, limitándose a anotar las otras cuestiones importantes, que pertenecen más bien al campo constitucional.

\subsection{Sentencia del Juzgado en lo Civil}

3.1.1. Sostiene que por tratarse de una acción de garantia, "no puede ser desestimada por el Juzgador sólo por el hecho de que los accionantes no han acreditado en'autos la representación que alegan", dado que en la Ley 23506 existe una disposición "que obliga al juzgador a suplir bajo responsabilidad cualquier deficiencia procesal en que incurra la parte reclamante".

3.1.2. Afirma que la acción de amparo y la acción popular son "vias paralelas", a mi modo de ver erróneamente conceptuadas como tales, dado que lo que constituyen vias paralelas son las acciones de garantía respecto de las ordinarias.

3.1.3. Pese a percibir que las normas que disponen la suspensión son decretos de urgencia, las asimila al nivel jerárquico de los decretos supremos, sosteniendo que "la Consti-

(25) Ojeda Avilés, Antonio, "La concurrencia de convenios colectivos", en Revista de Politica Social No 89 , Madrid. 
tución prohibe expresamente que un Decreto Supremo como el impugnado (de menor jerarquia) modifique o derogue una ley o pacto colectivo". He señalado antes que no es el rango de estas normas el argumento correcto para impedir su presencia en esta materia, sino su naturaleza no extraordinaria y su materia no económica y financiera.

3.1.4. Sostiene que los derechos violados "constituyen derechos adquiridos y por tanto irrenunciables" y que "el horario desde el punto de vista laboral es una condición de trabajo que no puede ni debe ser modificada en forma unilateral y compulsiva por el empleador" y declara fundada la demanda. Respecto de lo primero, he señalado que efectivamente hay en este caso derechos adquiridos, pero creo que en él no opera de modo alguno el principio de irrenunciabilidad, por cuanto no hay acto voluntario de privación de un derecho por los trabajadores.

Respecto de la segunda, no se percibe que el problema es de duración de la jornada y no de un simple cambio de horario (que sería atribución del empleador alterar siempre que no lo hiciera con el propósito de perjudicar al trabajador), así como que no hay modificación unilateral por el empleador, en tanto al dictar los decretos el Estado ha actuado como poder político y no como persona jurídica.

\subsection{Sentencia de la Corte Superior}

3.2.1. Arriba al mismo resultado que la primera respecto de la personería jurídica de la entidad accionante, pero por via distinta: considera que "está en autos suficientemente probada".

3.2.2. Pese a aceptar apparentemente que ha habido violación de un derecho, revoca la sentencia de primera instancia porque el reclamente "ha admiticlo tal variación y aumento de horas de servicios efectivos prestados, al someterse a tales decisiones", razón por la cual "ya no es posible "reponer las cosas al estado anterior', que constituye el objeto de las acciones de garantia". El punto primero lo considero -además de falso: hubo rebeldia frente a la medida-insostenible porque los trabajadores no puedan dejar de cumplir una nor- 
ma aun cuando la consideren inconstitucional hasta que el Poder Judicial o el Tribunal de Garant ias Constitucionales. en su caso, no lo declaren así. El segundo conlleva una interpretación restrictiva de las acciones de garantia, que perjudica a los accionantes, cuando si la reposición de las cosas al estado anterior fuera imposible, lo sería por la injustificable dilación del proceso (la Corte Superior resuelve el 1.7.86 una demanda que se habia interpuesto el 7.1.86 y resuelto en primera instancia el 27.1.86).

\subsection{Sentencia de la Corte Suprema}

3.3.1. Considera que, en cuanto la acción, persigue la inaplicabilidad de la medida para los afectados, está enmarcada dentro de los alcances de la acción de amparo reconocidos por el art. 295 de la Constitución.

3.3.2. Estima que no invalida el derecho de los accionantes "la circunstancia de no ser posible reponer las cosas al estado anterior tanto porque la cautela a que obliga el acotado dispositivo constitucional es perentorio y supera, por lo mismo, cualquier aparente limitación que pueda emanar de lo dispuesto por el art. 1 ro. de la Ley de Habeas Corpus y Amparo". En este aspecto, la Corte Suprema interpretu que el objeto de las acciones de garantía en la Ley 23506 es más restringido que en el art. 295 de la Constitución, por lo que - sin decirlo expresamente- en virtud de la mayor jerarquia de esta última disposición la prefiere sobre la primera.

3.3.3. Añade que "además no puede soslayarse que la dación posterior del Decreto Supremo 068-86-PCM de fecha 26.12.86, que repite en los mismos términos e impugnado número 107-85-PCM pone de manifiesto la vigencia del mismo estado de cosas que dieron lugar a la instauración de la materia de este proceso". Al respecto debo decir que, como lo sentencia se emite el 31.12.86, al dictarse ya se habia expedido el segundo decreto de urgencia, vigente para 1987, estableciéndose correctamente cl vínculo entre ambas medidas. Por tal razón, la Corte Suprema declara haber nulidad en la sentencia de vista y confirma la de primera instancia. 
3.3.4. En virtud de lo dispuesto por la propia Ley 23506 , esta sentencia genera un precedente obligatorio, que ya ha sido seguido por otras resoluciones en el mismo sentido dictadas en casos iguales y que debe desalentar la repetición futura de estos hechos, al menos por la vía de los decretos de urgencia.

\section{CONCLUSIONES}

4.1. Los trabajadores al servicio del Estado, tanto en las empresas estatales como en la administración pública, habían obtenido por vía de convenios colectivos y resoluciones administrativas en el primer caso y de leyes en el segundo, el derecho a laborar menos horas por día que lo ordinario durante los meses de verano. Tal derecho fue suspendido en 1986 y 1987 por sendos decretos de urgencia, que restablecieron la jormada de 8 horas.

4.2. Los decretos de urgencia son normas inapropiadas para adoptar dicha medida, porque exceden en este caso los supuestos que les permiten actuar válidamente: urgente y extraordinaria necesidad y materia económica y financiera. Por tal razón, resultan inconstitucionales.

4.3. El art. 44 de la Constitución referido a la jornada de trabajo establece una norma de derecho necesario relativo, que permite su mejoramicnto por ley o convenio colectivo. En caso de producirse éstas, constituirían nuevos mínimos infranqueables para disposiciones de menor rango o actos individuales de los trabajadores.

4.4. En el caso que he tratado hubo leyes y convenios colectivos que dispusieron la disminución de la jornada, que fueron vulnerados por decretos de urgencia. De haber sido una ley la que habría contenido la reampliación de la jornada. habría sido totalo parcialmente ineficaz. Respecto de la ley original, la hubiera derogado, pero tendría que respetar los derechos adquiridos, por lo que su alcance se restringiria a los nuevos trabajadores. Respecto del convenio colectivo original, se habria constituido en una norma inaplicable por su contenido peyorativo comparado con el de ą̧uél, en virtud del principio de norma más favorable. 